\title{
Epigenetic regulation mediated by microRNAs in the susceptibility and pathogenesis of rheumatoid arthritis
}

\author{
Shicheng Guo ${ }^{1,2 \#^{*}}$, Cen Chang ${ }^{3,4 \#}$, Lingxia $\mathrm{Xu}^{3,4 \#}$, Runrun Zhang ${ }^{3,4}$, Yehua $\mathrm{Jin}^{3,4}$, \\ Momiao Xiong ${ }^{5}$, and Dongyi $\mathrm{He}^{3,4,6^{*}}$
}

${ }^{1}$ Department of Medical Genetics, School of Medicine and Public Health, University of Wisconsin-Madison, Madison, WI, USA.

${ }^{2}$ Center for Precision Medicine Research, Marshfield Clinic Research Institute, Marshfield, WI, USA

${ }^{3}$ Shanghai University of Traditional Chinese Medicine, Shanghai, China

${ }^{4}$ Department of Rheumatology, Shanghai Guanghua Hospital, Shanghai University of Traditional Chinese Medicine, Shanghai, China.

${ }^{5}$ Department of Biostatistics and Data Science, School of Public Health 1200 Herman Pressler, University of Texas Health Science Center, Houston, USA.

${ }^{6}$ Arthritis Institute of Integrated Traditional and Western medicine, Shanghai Chinese Medicine Research Institute, Shanghai, China.

\#SG, CC, and LX contributed equally to the study.

\section{${ }^{*}$ Correspondence:}

Shicheng Guo, Ph.D.

Department of Medical Genetics

School of Medicine and Public Health

University of Wisconsin-Madison, Madison

Tel: 281-685-5882

Email: Shicheng.Guo@wisc.edu

Dongyi He, M.D., Ph.D.

Department of Rheumatology

Shanghai Guanghua Hospital, Shanghai University of Traditional Chinese Medicine

Shanghai, China

Tel : 158-0030-0800

Email: hedongyi1967@shutcm.edu.cn

Manuscript length: 2706 words; 1 Figure.

Running title: miRNA-mediated regulation of rheumatoid arthritis

Keywords: rheumatoid arthritis, miRNA, susceptibility, pathogenesis, epigenetics 


\begin{abstract}
MicroRNAs (miRNAs) play crucial roles in the regulation of the transcriptome and development of diseases including cancer and autoimmune diseases, such as rheumatoid arthritis (RA). Currently, a comprehensive map, illustrating how miRNAs regulate transcripts, pathways, immune system differentiation, and their interaction with terminal cells, such as T cells, fibroblast-like synoviocytes (FLS), osteoblasts, and osteoclasts, is still missing. In this review, we provide a thorough summary of the roles of miRNAs in the susceptibility to pathogenesis, diagnosis, therapeutic intervention, and prognosis of RA. Numerous miRNAs are abnormally expressed in cells involved in RA, and regulate target genes and pathways including the NF-KB, Fas-FasL, JAK-STAT, IRE1-RIDD, and mTOR pathways. By regulating gene expression, miRNAs affect $T$ cell differentiation to diverse cell types, including Th17 and T-reg cells, and thus constitute promising gene therapy targets to modulate the immune system in RA. We summarize the diagnostic and prognostic potential of blood-circulating and cell-free miRNAs, highlighting the novel opportunities to combine these with rheumatoid factor (RF) and anti-cyclic citrullinated peptide (anti-CCP) to provide accurate diagnosis and prognosis, especially for seronegative patients. Furthermore, we outline how functional genetic variants of miR-499 and miR-146a partly explain the unmet susceptibility to RA. Additionally, we review the evidence implicating miRNAs as promising biomarkers of efficiency, response, and resistance to disease-modifying anti-rheumatic drugs (DMRDs) and immunotherapy. Finally, we discuss the autotherapeutic effect of miRNA intervention as a step toward the development of miRNA-based anti-RA drugs. Collectively, the current evidence supports miRNAs as interesting targets to better understand the pathogenetic mechanisms of RA and design more efficient therapeutic interventions.
\end{abstract}

\title{
Introduction
}

Rheumatoid arthritis (RA) is an autoimmune disease characterized by chronic joint inflammation and structural damage, accompanied by extra-articular manifestations such as rheumatoid nodules, interstitial pneumonia, vasculitis, and systemic complications. RA is usually progressive and insidious, and the incidence rate is $0.5-1 \%$ in Europe and North America (Ibanez-Cabellos et al., 2019). However, the precise mechanisms underlying pathogenesis, disease activity, and severity of RA as well as the causes of different response to treatment are not fully understood. In view of the current therapy strategies and treatment frames, early accurate diagnosis, effective and personalized treatment, and precision medicine have become increasingly urgent for RA patients. A comprehensive understanding of RA is hence required, from the angles of both genetics (HLA and nonHLA variants) (Okada et al., 2014) epigenetics (DNA methylation (Guo et al., 2017; Chen et al., 2019; Guo et al., 2020b), microRNA (Furer et al., 2010; Mizoguchi and Kohsaka, 2012), IncRNA (Bi et al., 2019; Guo et al., 2019), and histone modifications (Angiolilli et al., 2017)).

MicroRNAs (miRNAs) are small endogenous non-coding single-stranded RNAs, with a length of about 22 nucleotides, which are involved in the post-transcriptional regulation of 
gene expression. In recent years, accumulating studies have demonstrated that miRNAs play a key role in various cancers (He et al., 2011; Lin et al., 2013; Zhang et al., 2015; Ding et al., 2016; Fan et al., 2017) as well as autoimmune diseases, including RA, systemic lupus erythematosus (SLE) (Xie and Xu, 2018; Senousy et al., 2019), sjogren's syndrome (SS) (Jang et al., 2019), and systemic sclerosis (Iwamoto et al., 2016). In this review, we systematically summarize the recent advances on the role of miRNAs in RA, with special emphasis on how the genetic variants and expression variations correlate to the susceptibility to and pathogenesis of RA, based on different inflammation-related cells, inflammatory cytokines, and inflammatory signaling pathways (Figure 1).

\section{Genetic variations in miRNAs explained missing susceptibility of rheumatoid arthritis.}

Genome-wide association studies have identified $>100$ genetic factors for RA. However, the reported genetic variants only explain $<40 \%$ of the overall heritability of RA, leaving the majority of the heritability unaccounted for, thus suggesting the need for more studies that employ different approaches and populations in order to identify the missing causes. Association studies to miRNA loci provided the opportunity to identify RA-associated functional or causal variants within different populations, such as Chinese (Yang et al., 2011; Yang et al., 2012), Egyptian (El-Shal et al., 2013; Ayeldeen et al., 2018; Shaker et al., 2018), Polish (Bogunia-Kubik et al., 2016), Mexican (Aleman-Avila et al., 2017), and Iranian (Hashemi et al., 2013). The rs3746444 (20q11.22, A>G) polymorphism of miR499, which is encoded by an intron of $M Y H 7 B$, is significantly linked to RA risk, disease activity, and methotrexate (MTX) toxicity (Toraih et al., 2016); interestingly, the AA genotype shows higher disease activity and MTX toxicity than AG/GG genotypes (Fattah et al., 2018). Gene expression and genetic polymorphisms of miR-146a and miR-499 showed diagnostic potential for RA (Ayeldeen et al., 2018).In addition, miR-146a rs2910164 is associated with RA susceptibility in the Egyptian population, in which the C allele is protective (Ayeldeen et al., 2018; Fattah et al., 2018). Consistently, the polymorphism rs3027898 in IRAK1, the target gene of miR-146a, is linked to RA in the Greek population (Chatzikyriakidou et al., 2010). However, follow-up studies showed inconsistent results in Polish (Bogunia-Kubik et al., 2016), Mexican (Aleman-Avila et al., 2017), and Chinese (Yang et al., 2011; Yang et al., 2012; Zhou et al., 2015b; Yang et al., 2017) populations. SNPs in other miRNAs were also tested in some studies, but their association with RA in other populations was not significant. For example, miR-196a-2 rs11614913 C/T and miR-499 rs3746444 A/G are not significantly associated with RA in Mexicans (Aleman-Avila et al., 2017), while miR-146a rs2910164 (Yang et al., 2011; Zhou et al., 2015b) and miR-499 rs3746444 (Yang et al., 2011; Yang et al., 2017) do not significantly correlate with RA in Chinese. In our recent study, we demonstrated that metaanalysis could identify more significant SNPs in a large sample size, and found that the interaction between HLA alleles and miRNA SNPs (such as rs5997893 in miR-3928 and rs4947332 in HLA-DRB1) should also be considered to explain unmet susceptibility (Guo et al., 2020a). In summary, genetic variations in miRNAs aids in explaining the missed susceptibility of rheumatoid arthritis.

\section{Genes and signaling pathways regulated by miRNA in the development of rheumatoid arthritis}

The cells involved in the pathogenesis of RA include CD4 ${ }^{+} \mathrm{T}$ cells, such as Th1, T-reg, and Th17 cells, as well as fibroblast-like synoviocyte (FLS), osteoclasts, and macrophages. The current research has mainly focused on understanding miRNA-mediated 
transcriptional regulation of FAF1 (Li et al., 2010), TNF- $\alpha$ (Trenkmann et al., 2013; Gao et al., 2018), STAT1 (Zhou et al., 2015a), STAT3 (Liu et al., 2017), TLR4 (Li et al., 2019a) and $m$ TOR (Tang et al., 2019; Zhu et al., 2020). miRNAs regulate inflammation, immune response, proliferation, differentiation, and influence of micro-environment within synovial joints by targeting these genes and their related pathways, including Fas-FasL (Li et al., 2010) and NF-kB (Stanczyk et al., 2011; Trenkmann et al., 2013; Wang et al., 2019a) pathway. In this section, we summarize the regulatory roles of miRNAs in the main RAassociated cell entities, focusing on T cells, FLS, and osteoclasts, to highlight the importance of miRNAs in the pathogenesis of RA.

\section{miRNA-mediated innate and adaptive immune cell differentiation in rheumatoid arthritis}

The balance of T-reg/Th17 cells plays a crucial role in RA. IL-17 released by Th17 upregulates expression of receptor activator of nuclear factor-kB ligand (RANKL) on synovial fibroblasts and stimulates production of inflammatory cytokines such as TNF- $\alpha$, IL-6, and IL- 1 by immune cells (van Hamburg and Tas, 2018). The regulatory roles of miR-146a have been widely studied in T cells. The expression of miR-146a is significantly increased in $\mathrm{CD}^{+} \mathrm{T}$ cells, peripheral blood mononuclear cells (PBMCs), and Jurkat $\mathrm{T}$ cells, promoting T cell differentiation and inhibiting apoptosis (Li et al., 2010; Niimoto et al., 2010). Interestingly, the expression of miR-146a decreases in T-reg cells during high RA activity, leading to a proinflammatory phenotype in these cells caused by concomitant upregulation of its target STAT1 (Zhou et al., 2015a). High levels of miR-99b-5p (Zhu et al., 2020), miR-361-5p (Romo-Garcia et al., 2019), and miR-17 (Wang et al., 2018a) contribute to enhanced $\mathrm{T}$ cell proliferation and differentiation, and inhibit apoptosis. In PBMCs, miR-99b-5p down-regulates $m T O R$ and RASSF4 genes, thereby inhibiting T cell apoptosis, and promoting T cell proliferation and inflammatory response (Zhu et al., 2020). Up-regulation of miR-17 in RA exosomes inhibits the differentiation of T-reg by suppressing the expression of TGFB2 (Wang et al., 2018a). Interestingly, miR-21 level is decreased in circulating PBMCs (Dong et al., 2014) but increased in Vү9Vठ2T cells (Guggino et al., 2018) in RA patients, which could be associated with the imbalance of Th17/T-reg cells. Although miR-120-mediated negative regulation of $H I F-1$ also affects the dynamic equilibrium of Th17/T-reg, no association has been found between this miRNA and RA (Huang et al., 2018).

MiRNA-induced DNA methylation, in addition to T cell differentiation, is also critical for the pathogenesis of RA. For example, miR-126 inhibits methylation of the promoter region in CD70 and CD11a, thereby promoting their expression (Yang et al., 2015). In macrophages, binding of miR-6089 and IncRNA-HIX003209 enhances the expression of TLR4 and exacerbates inflammation through the TLR4/NF-kB pathway (Yan et al., 2019). In addition, miR-30a can increases inflammation, by aggravating autophagy and reducing apoptosis (Xu et al., 2013). Over-expression of miR-192-5p ameliorates the inflammatory response in RA by targeting the up-regulation of ras-related c3 botulinum toxin substrate 2 (RAC2) (Zheng et al., 2020). Increasing the expression of miR-20 and miR-211 down-regulates their target ATF2, thereby reducing inflammation in RA model cells SW982 (Xie et al., 2018). The decreased expression of miR-671 and miR-96 in PBMCs of patients with RA may correlate with expression of mTOR (Tang et al., 2019) and endoplasmic reticulum stress induced by inositol-requiring enzyme 1 alpha (IRE1)/endoplasmic reticulum stress (RIDD) pathway (Ahmadiany et al., 2019). In PBMCs, miR-29b enhances the antiapoptotic effect by inhibiting HMG-box transcription factor 1 (HBP1) pathway (Ren et al., 2019). Evidence shows that $m i R-198, m i R-4647$, and $m i R-7167-5 p$ are also connected to 
T cell signaling, apoptosis, and immune response (Raj Christian et al., 2019). Numerous other miRNAs are associated with the T-reg subpopulations, such as miR-21 and miR155 that are related to the memory phenotype, or miR-92a that is related to the naive phenotype (Smigielska-Czepiel et al., 2014). The expression of $m i R-223$ is high in naive $\mathrm{CD}^{+} \mathrm{T}$ cell, but hardly expressed in Th17 (Fulci et al., 2010). Overexpression of miR-361$5 p$ in early RA is associated with $T$ cell activation and inflammatory response (RomoGarcia et al., 2019). Overall, miRNAs cooperate with other non-coding RNAs (ncRNAs) to alter the DNA methylation and/or expression of their targets, thus regulating innate and adaptive immune cell differentiation and apoptosis, and ultimately influencing the inflammatory and autoimmune response in RA.

\section{miRNA-mediated cell differentiation of fibroblast-like synoviocytes in rheumatoid arthritis}

Synovial fibroblasts are key regulators of inflammation and bone destruction in RA. In addition to producing RANKL, fibroblast-like synoviocytes in RA (RA-FLS) also activate osteoclast differentiation by releasing inflammatory cytokines, chemokines, and matrix metalloproteinases (MMPs) (Rana et al., 2018). Ample evidence shows that downregulation of miR-22 (Lin et al., 2014), miR-29c-3p (Tseng et al., 2019), miR-124a (Nakamachi et al., 2009), and miR-4701-5p (Bi et al., 2019), and up-regulation of miR-143 (Hong et al., 2017), miR-145 (Hong et al., 2017), and miR-191 (Yu et al., 2019) enhances the proliferation, migration, and invasion of RA-FLS. In contrast, down-regulation of miR132-3p (Tseng et al., 2019) and miR-29a (Liu et al., 2017), and up-regulation of miR-31$5 p$ (Tseng et al., 2019) and miR-124a (Meng et al., 2020) inhibits these processes (Tseng et al., 2019). Besides, miR-199a-3p (Wangyang et al., 2018), miR-449a (Cai et al., 2019), miR-506 (Li et al., 2019a), and miR-126 (Gao et al., 2018), whose expression is decreased in RA, inhibit RA-FLS proliferation and induce apoptosis by targeting $R B 1$, high-mobility group box protein 1 (HMGB1), TLR4, and IL-23R, respectively. Proliferation and invasion of RA-FLS is also correlated with MMP (Tolboom et al., 2002). In RA-FLS, up-regulation of miR-145-5p (Wang et al., 2019a), miR-18a (Trenkmann et al., 2013), miR-155 (Stanczyk et al., 2008; Long et al., 2013), and miR-203 (Stanczyk et al., 2011), and downregulation of $\mathrm{miR}-27 \mathrm{a}$ (Shi et al., 2016) contribute to MMP expression by targeting the NFKB (Stanczyk et al., 2011; Trenkmann et al., 2013; Wang et al., 2019a), TLR4 (Oka et al., 2017), and Follistatin like-1 (FSTL1) (Shi et al., 2016) pathway. What's more, miRNAs can also regulate specific genes related to RA-FLS phenotypic differentiation. For example, miR-625 is down-regulated in RA-FLS, which negatively impacts on expression of CTSC, $K L F 8, E B F 3$; instead, miR-551b is up-regulated in RA-FLS, inhibiting expression of ITGBL1 (de la Rica et al., 2013). In RA, bone loss is mainly due to overabsorption of bone by osteoclasts and weakened osteoblast bone formation (Okamoto et al., 2017).In vitro, overexpression of miR-221-3p inhibits osteoblast differentiation (Maeda et al., 2017). Instead, miR-218 overexpression promotes osteogenic differentiation of RA-FLS by suppressing the Roundabout-1 (ROBO1)/Dickkopf-1 (DKK1) axis (Iwamoto et al., 2018). In summary, miRNAs are widely involved in cell differentiation of fibroblast-like synoviocytes and osteoblast and therefore may be promising targets for drug development and enhance our understanding to the pathogenesis of rheumatoid arthritis.

\section{Blood and serum-circulating miRNAs provide novel opportunities for the diagnosis of rheumatoid arthritis}

Emerging evidence points out the potential of blood-circulating miRNAs associated with RA as potential biomarkers for diagnosis, prognosis, as well as disease activity. The levels 
of $m i R-371 b, m i R-483$, and $m i R-642 b$ are significantly up-regulated while $m i R-25$ and miR-378d are down-regulated in PBMCs in individuals that eventually develop RA from early undifferentiated arthritis (Kurowska et al., 2018). Meanwhile, miR-22 (Ouboussad et al., 2017), miR-361-5p (Romo-Garcia et al., 2019), and miR-223-3p (Romo-Garcia et al., 2019) are significantly up-regulated in high-risk or CCP-positive populations. All these miRNAs could therefore be used as biomarkers for early diagnosis of RA. Expression of $m i R-103 a-3 p$ is significantly increased in autoantibody-positive, symptomatic first-degree relatives (FDR) and RA patients, suggesting it as a potential biomarker for predicting imminent disease in individuals at risk for developing RA (Anaparti et al., 2017). Additionally, higher levels of miR-143-3p, miR-145-5p, and miR-99b-5p are found in the plasma of early RA patients with bone erosion, indicating that they could be monitored for bone erosion surveillance in RA patients. Furthermore, miR-99b-5p is demonstrated to be an independent predictor of bone erosion progression in early RA (Yue et al., 2019).

In addition to playing a role in early recognition of RA, the expression of some miRNAs aids to diagnose RA with higher accuracy (Evangelatos et al., 2019). Expression of miR$146 a$ and miR-155 are significantly increased in RA PBMCs, and shows a similar trend in whole blood (Mookherjee and El-Gabalawy, 2013). The levels of miR-24 and miR-125a are significantly higher in the serum of RA patients regardless of CCP status (Murata et al., 2013). Interestingly, analysis of $m i R-24-3 p$, miR-26a-5p, and miR-125a-5p levels in combination constitutes a better diagnostic tool for RA, even though these miRNAs are not related to disease activity (Ormseth et al., 2015). What's more, miR-122-3p, miR-3925$3 p$, miR-342-3p, and miR-4764-5p show differential expression not only between healthy individuals and RA patients, but also between RA patients and osteoarthritis (OA), SLE, or Graves patients (Wang et al., 2015). Other differentially expressed miRNAs in RA patients serum include miR-4634, miR-181d, miR-3926, miR-3926, miR-9-5p, miR-219-23p6, miR-221, miR-222, miR-532, miR-106a, and miR-987, which highlights their potential as RA-specific diagnostic markers (Wang et al., 2015; Khalifa et al., 2016).

The serum levels of miR-146a (Abou-Zeid et al., 2011), miR-22-3p (Ormseth et al., 2020a), miR-5571-3p (Liu et al., 2019a), and miR-135b-5p (Liu et al., 2019a) are significantly higher in RA patients than in healthy controls and OA patients. Additionally, in RA patients, the expression of miR-451 in $\mathrm{T}$ cells is significantly increased, which is positively correlated with the levels of disease activity score 28 (DAS28), erythrocyte sedimentation rate (ESR) and serum IL-6 (Smigielska-Czepiel et al., 2014). The level of miR-146a is positively correlates with the level of ESR and DAS28 (Abou-Zeid et al., 2011), while miR5571-3p (Liu et al., 2019a) correlates with the level of ESR and C-reactive protein (CRP), and miR-135b-5p only with CRP (Liu et al., 2019a). These miRNAs could therefore be suitable markers of disease activity in RA patients.

Increase in serum miR-194-5p levels is associated with disease recurrence (FernandezRuiz et al., 2018). Serum expression of miR-23b, which positively correlates with ESR, CRP, and DAS28, is significantly up-regulated after appropriate treatment, indicating that miR-23b is a dual marker of disease activity and prognosis (Liu et al., 2019b). Similarly, miR-96-5p, miR-134-5p, miR-140-3p, and miR-627-5p are not only diagnostic markers for $\mathrm{RA}$, but also reflect disease activity (Ormseth et al., 2020b). In summary, the changes in miRNA levels in the serum of RA patients provide a promising opportunity for early diagnosis, as well as indication of disease activity and prediction of RA outcomes.

miRNAs as potential biomarkers for pharmacogenetics, therapeutic outcome, and treatment response prediction 
Common and widely used anti-rheumatic drugs include cDMARD (MTX, sulfasalazine, and hydroxychloroquine), bDMARD (TNF inhibitors, Rituximab, and Tocilizumab), and tsDMARD (Tofacitinib, Barretinib, and Filgotinib). Several studies have explored the relationship between serum miRNA levels and the response to DMARD. Evidence shows that high serum level of miR-10 in RA patients correlate with good response to MTX (Hong et al., 2018). After 3 months of adalimumab (ADA)/MTX combined treatment, serum level of $m i R-27 a-3 p$ significantly decrease and the clinical symptoms of RA show remission (Sode et al., 2018). Serum level of miR-5196 also significantly decrease in RA and ankylosing spondylitis (AS) patients after anti-TNF- $\alpha$ therapy, and indicate lower DAS28 (Ciechomska et al., 2018). Meanwhile, serum levels of miR-146a diminish in RA patients who respond well to anti-TNF therapy, and can interestingly be considered as predictors of the response to anti-TNF- $\alpha$ therapy together with CRP (Castro-Villegas et al., 2015; Bogunia-Kubik et al., 2016; Liu et al., 2019c). By contrast, serum levels of miR-23 and miR-223 are increased in RA patients who respond well to anti-TNF-a/DMARD combination therapy, but correlate negatively to the response to anti-TNF drugs (CastroVillegas et al., 2015). High serum level of miR-125b is potential indicator of good clinical response to Rituximab therapy (Duroux-Richard et al., 2014). Notably, miR-432-5p is significantly down-regulated in RA patients who are responsive to Tofacitinib therapy, but up-regulated in RA relapse patients (Fernandez-Ruiz et al., 2018). In RA, treatment with Rituximab increases the levels of $m i R-16-5 p$ and $m i R-23 a-3 p$ in peripheral blood (PerezSanchez et al., 2019). The levels of $m i R-425-5 p$, miR-21-5p, and miR-212-3p significantly decrease in RA patients treated with glucocorticoids, although no clinical response studies have been conducted (Balzano et al., 2017).

In addition to DMARD treatment, alternative and complementary medicine preparations and mesenchymal stem cell (MSC) treatments are also used in the clinical treatment of RA. The expression of miR-550b-2-5p, miR-4797-5p, miR-6509-5p, miR-378g, miR-4720$5 p$, miR-374b-5p, and miR-185-3p are found differently between individuals who have good and poor response to treatment with tripterysium glycosides (TG) (Zhang et al., 2018; Wang et al., 2019b). Furthermore, miR-26b-5p, miR-487b-3p, and miR-495-3p are significantly up-regulated in the responders to adipose-derived mesenchymal stem cell treatment (Mallinson et al., 2017). Finally, miR-124a level in FLS increase following geniposide treatment; however, the relevance of this finding has not been assessed in clinical response studies (Wang et al., 2018b).

The auto-therapeutic effect of miRNAs has been demonstrated in mouse models of RA synovial fibroblasts (RASF) and autoimmune arthritis. For example, miR-506 mimics reduce the proliferation of RA-FLS and production of pro-inflammatory cytokines, while also promoting the apoptosis of RA-FLS (Li et al., 2019a), miR-449a mimics also inhibit proliferation, migration, and IL-6 production of RA-FLS by regulating HMGB1 and YY1 expression (Cai et al., 2019). In a rat model of collagen-induced arthritis (CIA), miR-708$5 p$ mimic improved the pathological changes by inhibiting inflammatory cell infiltration, synovial hyperplasia, and cartilage destruction (Wu et al., 2018), miR-126 agonist inhibits the expression of IL-23R, TNF- $\alpha$, and IFN-y in the FLS (Gao et al., 2018). MSC-derived miR-124a exosomes inhibit proliferation and migration and promote apoptosis of FLS cell lines (Meng et al., 2020). In addition, exo-miR-150 has been shown to inhibit RA-FLS proliferation and angiogenesis and reduce RA joint destruction by targeting MMP14 and VEGF in rat RASF and CIA models (Chen et al., 2018). In conclusion, miRNAs have important and demonstrated roles in the treatment of RA, and could represent not only promising outcome biomarkers, but also novel drug targets to decrease the severity of the disease. 


\section{miRNA research in rheumatoid arthritis: remaining challenges and future opportunities}

MiRNAs play multiple roles in the development of RA, from susceptibility to pathogenesis. Blood and serum-circulating miRNAs have been explored as important biomarkers for the early diagnosis, prognosis, as well as drug response prediction. Furthermore, miRNAs have been proposed for autotherapeutic approaches and as novel drug targets for the treatment of RA. Genetic variants in specific miRNAs can increase or decrease the risk and disease activity of RA in different ethnicities, and are associated with methotrexate toxicity and responses to other treatments. Moreover, the changes in miRNAs in various cells are related to the pathogenesis of and pathological changes occurring in RA, such as proliferation and differentiation of immune cells, proliferation and apoptosis of synovial cells, and synovial inflammation and cartilage destruction. Research has remarkably progressed towards the development of miRNAs as biomarkers for the diagnosis, prognosis, disease activity, and response to therapeutic drugs with RA, providing a direction for early diagnosis and accurate treatment of RA, to ultimately achieve better treatment efficiency and precision medicine in the near future. Numerous miRNAs have been shown to act as therapeutic targets in RA-FLS and CIA rat models. MiRNAs also showed the promising ability to identify subtypes of RA: for example, the expression levels of $m i R-7$ and miR-214-5p are significantly increased in the serum of RA associatedinterstitial lung disease (RA-ILD) patients (Oka et al., 2017), while miR-9-5p targets REST/miR-132 pathway to protect Schwann cells from inflammatory damage in RAinduced peripheral neuropathy ( $\mathrm{Li}$ et al., 2019b). Although we have reached exciting milestones in the research on the multiple roles of miRNA in RA, more relevant studies should be implemented to understand and transfer the available knowledge into the clinical application, but also solve the current inconsistent results among different studies employing different methods or populations. For example, studies on miR-99, miR-143, and $m i R-197$ as the landmark miRNAs to predict the response to anti-TNF- $\alpha$ therapy have failed to yield results consistent with those previously reported (Cuppen et al., 2016). Finally, we expect the future development of miRNA-based baseline RA polygenetic risk score models, especially in conjunction with HLA. Meanwhile, miRNA-based early diagnosis, prognosis, and drug response prediction models could be applied in future clinical applications. Hopefully, with the identification of more miRNA-based drug targets in clinical research, miRNA-based autotherapeutic treatments could show more promising results.

\section{Conflict of Interest}

The authors declare that the research was conducted in the absence of any commercial or financial relationships that could be construed as a potential conflict of interest.

\section{Author contributions}

SG and DH conceived the content. SG, CC, and LX wrote the manuscript, RZ, YJ, and $\mathrm{MX}$ edited the manuscript. All authors read and approved the final manuscript.

\section{Acknowledgments}

This work was funded by the National Natural Science Funds of China (81774114), Shanghai Chinese Medicine Development Office, Shanghai Chinese and Western Medicine Clinical Pilot Project (ZY (2018-2020)-FWTX-1010), Shanghai Chinese 
Medicine Development Office, Shanghai Traditional Chinese Medicine Specialty Alliance Project (ZY (2018-2020)-FWTX-4017), National Administration of Traditional Chinese Medicine, Regional Chinese Medicine (Specialist) Diagnosis and Treatment Center Construction Project-Rheumatology. The manuscript have previously appeared online, doi:10.20944/preprints202004.0241.v1

\section{References}

Abou-Zeid, A., Saad, M., and Soliman, E. (2011). MicroRNA 146a expression in rheumatoid arthritis: association with tumor necrosis factor-alpha and disease activity. Genet Test Mol Biomarkers 15 (11), 807-812. doi: 10.1089/gtmb.2011.0026.

Ahmadiany, M., Alavi-Samani, M., Hashemi, Z., Moosavi, M.A., and Rahmati, M. (2019). The Increased RNase Activity of IRE1alpha in PBMCs from Patients with Rheumatoid Arthritis. Adv Pharm Bull 9 (3), 505-509. doi: 10.15171/apb.2019.060.

Aleman-Avila, I., Jimenez-Morales, M., Beltran-Ramirez, O., Barbosa-Cobos, R.E., Jimenez-Morales, S., Sanchez-Munoz, F., et al. (2017). Functional polymorphisms in pre-miR146a and pre-miR499 are associated with systemic lupus erythematosus but not with rheumatoid arthritis or Graves' disease in Mexican patients. Oncotarget 8 (54), 91876-91886. doi: 10.18632/oncotarget.19621.

Anaparti, V., Smolik, I., Meng, X., Spicer, V., Mookherjee, N., and El-Gabalawy, H. (2017). Whole blood microRNA expression pattern differentiates patients with rheumatoid arthritis, their seropositive first-degree relatives, and healthy unrelated control subjects. Arthritis Res Ther 19 (1), 249. doi: 10.1186/s13075-017-1459-x.

Angiolilli, C., Kabala, P.A., Grabiec, A.M., Van Baarsen, I.M., Ferguson, B.S., Garcia, S., et al. (2017). Histone deacetylase 3 regulates the inflammatory gene expression programme of rheumatoid arthritis fibroblast-like synoviocytes. Ann Rheum Dis 76 (1), 277-285. doi: 10.1136/annrheumdis2015-209064.

Ayeldeen, G., Nassar, Y., Ahmed, H., Shaker, O., and Gheita, T. (2018). Possible use of miRNAs-146a and -499 expression and their polymorphisms as diagnostic markers for rheumatoid arthritis. Mol Cell Biochem 449 (1-2), 145-156. doi: 10.1007/s11010-018-3351-7.

Balzano, F., Deiana, M., Dei Giudici, S., Oggiano, A., Pasella, S., Pinna, S., et al. (2017). MicroRNA Expression Analysis of Centenarians and Rheumatoid Arthritis Patients Reveals a Common Expression Pattern. Int J Med Sci 14 (7), 622-628. doi: 10.7150/ijms.18972.

Bi, X., Guo, X.H., Mo, B.Y., Wang, M.L., Luo, X.Q., Chen, Y.X., et al. (2019). LncRNA PICSAR promotes cell proliferation, migration and invasion of fibroblast-like synoviocytes by sponging miRNA-4701-5p in rheumatoid arthritis. EBioMedicine 50, 408-420. doi:

10.1016/j.ebiom.2019.11.024.

Bogunia-Kubik, K., Wysoczanska, B., Piatek, D., Iwaszko, M., Ciechomska, M., and Swierkot, J. (2016). Significance of Polymorphism and Expression of miR-146a and NFkB1 Genetic Variants in Patients with Rheumatoid Arthritis. Arch Immunol Ther Exp (Warsz) 64 (Suppl 1), 131-136. doi: 10.1007/s00005-016-0443-5.

Cai, Y., Jiang, C., Zhu, J., Xu, K., Ren, X., Xu, L., et al. (2019). miR-449a inhibits cell proliferation, migration, and inflammation by regulating high-mobility group box protein 1 and forms a mutual inhibition loop with Yin Yang 1 in rheumatoid arthritis fibroblast-like synoviocytes. Arthritis Res Ther 21 (1), 134. doi: 10.1186/s13075-019-1920-0.

Castro-Villegas, C., Perez-Sanchez, C., Escudero, A., Filipescu, I., Verdu, M., Ruiz-Limon, P., et al. (2015). Circulating miRNAs as potential biomarkers of therapy effectiveness in rheumatoid arthritis patients treated with anti-TNFalpha. Arthritis Res Ther 17, 49. doi: 10.1186/s13075-0150555-z. 
Chatzikyriakidou, A., Voulgari, P.V., Georgiou, I., and Drosos, A.A. (2010). A polymorphism in the 3'UTR of interleukin-1 receptor-associated kinase (IRAK1), a target gene of miR-146a, is associated with rheumatoid arthritis susceptibility. Joint Bone Spine 77 (5), 411-413. doi: 10.1016/j.jbspin.2010.05.013.

Chen, S., Pu, W., Guo, S., Jin, L., He, D., and Wang, J. (2019). Genome-Wide DNA Methylation Profiles Reveal Common Epigenetic Patterns of Interferon-Related Genes in Multiple Autoimmune Diseases. Front Genet 10, 223. doi: 10.3389/fgene.2019.00223.

Chen, Z., Wang, H., Xia, Y., Yan, F., and Lu, Y. (2018). Therapeutic Potential of Mesenchymal CellDerived miRNA-150-5p-Expressing Exosomes in Rheumatoid Arthritis Mediated by the Modulation of MMP14 and VEGF. J Immunol 201 (8), 2472-2482. doi: 10.4049/jimmunol.1800304.

Ciechomska, M., Bonek, K., Merdas, M., Zarecki, P., Swierkot, J., Gluszko, P., et al. (2018). Changes in MiRNA-5196 Expression as a Potential Biomarker of Anti-TNF-alpha Therapy in Rheumatoid Arthritis and Ankylosing Spondylitis Patients. Arch Immunol Ther Exp (Warsz) 66 (5), 389-397. doi: 10.1007/s00005-018-0513-y.

Cuppen, B.V., Rossato, M., Fritsch-Stork, R.D., Concepcion, A.N., Schenk, Y., Bijlsma, J.W., et al. (2016). Can baseline serum microRNAs predict response to TNF-alpha inhibitors in rheumatoid arthritis? Arthritis Res Ther 18, 189. doi: 10.1186/s13075-016-1085-z.

de la Rica, L., Urquiza, J.M., Gomez-Cabrero, D., Islam, A.B., Lopez-Bigas, N., Tegner, J., et al. (2013). Identification of novel markers in rheumatoid arthritis through integrated analysis of DNA methylation and microRNA expression. J Autoimmun 41, 6-16. doi: 10.1016/j.jaut.2012.12.005.

Ding, H., Gao, G., Zhang, L., Shen, G., Sun, W., Gu, Z., et al. (2016). The protective effects of curculigoside A on adjuvant-induced arthritis by inhibiting NF-small ka, CyrillicB/NLRP3 activation in rats. Int Immunopharmacol 30, 43-49. doi: 10.1016/j.intimp.2015.11.026.

Dong, L., Wang, X., Tan, J., Li, H., Qian, W., Chen, J., et al. (2014). Decreased expression of microRNA21 correlates with the imbalance of Th17 and Treg cells in patients with rheumatoid arthritis. $J$ Cell Mol Med 18 (11), 2213-2224. doi: 10.1111/jcmm.12353.

Duroux-Richard, I., Pers, Y.M., Fabre, S., Ammari, M., Baeten, D., Cartron, G., et al. (2014). Circulating miRNA-125b is a potential biomarker predicting response to rituximab in rheumatoid arthritis. Mediators Inflamm 2014, 342524. doi: 10.1155/2014/342524.

El-Shal, A.S., Aly, N.M., Galil, S.M., Moustafa, M.A., and Kandel, W.A. (2013). Association of microRNAs genes polymorphisms with rheumatoid arthritis in Egyptian female patients. Joint Bone Spine 80 (6), 626-631. doi: 10.1016/j.jbspin.2013.03.005.

Evangelatos, G., Fragoulis, G.E., Koulouri, V., and Lambrou, G.I. (2019). MicroRNAs in rheumatoid arthritis: From pathogenesis to clinical impact. Autoimmun Rev 18 (11), 102391. doi: 10.1016/j.autrev.2019.102391.

Fan, L., Chen, L., Ni, X., Guo, S., Zhou, Y., Wang, C., et al. (2017). Genetic variant of miR-4293 rs12220909 is associated with susceptibility to non-small cell lung cancer in a Chinese Han population. PLoS One 12 (4), e0175666. doi: 10.1371/journal.pone.0175666.

Fattah, S.A., Ghattas, M.H., Saleh, S.M., and Abo-Elmatty, D.M. (2018). Pre-micro RNA-499 Gene Polymorphism rs3746444 T/C is Associated with Susceptibility to Rheumatoid Arthritis in Egyptian Population. Indian J Clin Biochem 33 (1), 96-101. doi: 10.1007/s12291-017-0652-7.

Fernandez-Ruiz, J.C., Ramos-Remus, C., Sanchez-Corona, J., Castillo-Ortiz, J.D., Castaneda-Sanchez, J.J., Bastian, Y., et al. (2018). Analysis of miRNA expression in patients with rheumatoid arthritis during remission and relapse after a 5-year trial of tofacitinib treatment. Int Immunopharmacol 63, 35-42. doi: 10.1016/j.intimp.2018.07.028.

Fulci, V., Scappucci, G., Sebastiani, G.D., Giannitti, C., Franceschini, D., Meloni, F., et al. (2010). miR223 is overexpressed in T-lymphocytes of patients affected by rheumatoid arthritis. Hum Immunol 71 (2), 206-211. doi: 10.1016/j.humimm.2009.11.008. 
Furer, V., Greenberg, J.D., Attur, M., Abramson, S.B., and Pillinger, M.H. (2010). The role of microRNA in rheumatoid arthritis and other autoimmune diseases. Clin Immunol 136 (1), 1-15. doi: 10.1016/j.clim.2010.02.005.

Gao, J., Kong, R., Zhou, X., Ji, L., Zhang, J., and Zhao, D. (2018). MiRNA-126 expression inhibits IL23R mediated TNF-alpha or IFN-gamma production in fibroblast-like synoviocytes in a mice model of collagen-induced rheumatoid arthritis. Apoptosis 23 (11-12), 607-615. doi: 10.1007/s10495-018-1474-7.

Guggino, G., Orlando, V., Saieva, L., Ruscitti, P., Cipriani, P., La Manna, M.P., et al. (2018). Downregulation of miRNA17-92 cluster marks Vgamma9Vdelta2 T cells from patients with rheumatoid arthritis. Arthritis Res Ther 20 (1), 236. doi: 10.1186/s13075-018-1740-7.

Guo, S., J , Y., Zhou, J., Zhu, Q., Jiang, T., Bian, Y., et al. (2020a). MicroRNA variants and HLA-miRNA interactions are novel rheumatoid arthritis susceptibility factors. Annals of the Rheumatic Disease.

Guo, S., Liu, J., Jiang, T., Lee, D., Wang, R., Zhou, X., et al. (2019). (5R)-5-Hydroxytriptolide (LLDT-8) induces substantial epigenetic mediated immune response network changes in fibroblast-like synoviocytes from rheumatoid arthritis patients. Sci Rep 9 (1), 11155. doi: 10.1038/s41598-01947411-1.

Guo, S., Xu, L., Chang, C., Zhang, R., Jin, Y., and He, D. (2020b). Epigenetic regulation mediated by methylation in the pathogenesis and precision medicine of rheumatoid arthritis. Preprints. doi: 10.20944 / preprints202004.0237.v1.

Guo, S., Zhu, Q., Jiang, T., Wang, R., Shen, Y., Zhu, X., et al. (2017). Genome-wide DNA methylation patterns in CD4+ T cells from Chinese Han patients with rheumatoid arthritis. Mod Rheumatol 27 (3), 441-447. doi: 10.1080/14397595.2016.1218595.

Hashemi, M., Eskandari-Nasab, E., Zakeri, Z., Atabaki, M., Bahari, G., Jahantigh, M., et al. (2013). Association of pre-miRNA-146a rs2910164 and premiRNA-499 rs3746444 polymorphisms and susceptibility to rheumatoid arthritis. Mol Med Rep 7 (1), 287-291. doi: 10.3892/mmr.2012.1176.

He, Y., Cui, Y., Wang, W., Gu, J., Guo, S., Ma, K., et al. (2011). Hypomethylation of the hsa-miR-191 locus causes high expression of hsa-mir-191 and promotes the epithelial-to-mesenchymal transition in hepatocellular carcinoma. Neoplasia 13 (9), 841-853. doi: 10.1593/neo.11698.

Hong, B.K., You, S., Yoo, S.A., Park, D., Hwang, D., Cho, C.S., et al. (2017). MicroRNA-143 and -145 modulate the phenotype of synovial fibroblasts in rheumatoid arthritis. Exp Mol Med 49 (8), e363. doi: 10.1038/emm.2017.108.

Hong, H., Yang, H., and Xia, Y. (2018). Circulating miR-10a as Predictor of Therapy Response in Rheumatoid Arthritis Patients Treated with Methotrexate. Curr Pharm Biotechnol 19 (1), 79-86. doi: 10.2174/1389201019666180417155140.

Huang, Q., Chen, S.S., Li, J., Tao, S.S., Wang, M., Leng, R.X., et al. (2018). miR-210 expression in PBMCs from patients with systemic lupus erythematosus and rheumatoid arthritis. Ir J Med Sci 187 (1), 243-249. doi: 10.1007/s11845-017-1634-8.

Ibanez-Cabellos, J.S., Seco-Cervera, M., Osca-Verdegal, R., Pallardo, F.V., and Garcia-Gimenez, J.L. (2019). Epigenetic Regulation in the Pathogenesis of Sjogren Syndrome and Rheumatoid Arthritis. Front Genet 10, 1104. doi: 10.3389/fgene.2019.01104.

Iwamoto, N., Fukui, S., Takatani, A., Shimizu, T., Umeda, M., Nishino, A., et al. (2018). Osteogenic differentiation of fibroblast-like synovial cells in rheumatoid arthritis is induced by microRNA218 through a ROBO/Slit pathway. Arthritis Res Ther 20 (1), 189. doi: 10.1186/s13075-018-1703z.

Iwamoto, N., Vettori, S., Maurer, B., Brock, M., Pachera, E., Jungel, A., et al. (2016). Downregulation of miR-193b in systemic sclerosis regulates the proliferative vasculopathy by urokinase-type plasminogen activator expression. Ann Rheum Dis 75 (1), 303-310. doi: 10.1136/annrheumdis2014-205326. 
Jang, S.I., Tandon, M., Teos, L., Zheng, C., Warner, B.M., and Alevizos, I. (2019). Dual function of miR1248 links interferon induction and calcium signaling defects in Sjogren's syndrome. EBioMedicine 48, 526-538. doi: 10.1016/j.ebiom.2019.09.010.

Khalifa, O., Pers, Y.M., Ferreira, R., Senechal, A., Jorgensen, C., Apparailly, F., et al. (2016). X-Linked miRNAs Associated with Gender Differences in Rheumatoid Arthritis. Int J Mol Sci 17 (11). doi: 10.3390/ijms17111852.

Kurowska, W., Kuca-Warnawin, E., Radzikowska, A., Jakubaszek, M., Maslinska, M., Kwiatkowska, B., et al. (2018). Monocyte-related biomarkers of rheumatoid arthritis development in undifferentiated arthritis patients - a pilot study. Reumatologia 56 (1), 10-16. doi: 10.5114/reum.2018.74742.

Li, D., Zhou, Q., Hu, G., and Wang, G. (2019a). MiRNA-506 inhibits rheumatoid arthritis fibroblast-like synoviocytes proliferation and induces apoptosis by targetting TLR4. Biosci Rep 39 (5). doi: 10.1042/BSR20182500.

Li, J., Wan, Y., Guo, Q., Zou, L., Zhang, J., Fang, Y., et al. (2010). Altered microRNA expression profile with miR-146a upregulation in CD4+ T cells from patients with rheumatoid arthritis. Arthritis Res Ther 12 (3), R81. doi: 10.1186/ar3006.

Li, Z., Li, Y., Li, Q., Zhang, Z., Jiang, L., and Li, X. (2019b). Role of miR-9-5p in preventing peripheral neuropathy in patients with rheumatoid arthritis by targeting REST/miR-132 pathway. In Vitro Cell Dev Biol Anim 55 (1), 52-61. doi: 10.1007/s11626-018-0310-2.

Lin, J., Huo, R., Xiao, L., Zhu, X., Xie, J., Sun, S., et al. (2014). A novel p53/microRNA-22/Cyr61 axis in synovial cells regulates inflammation in rheumatoid arthritis. Arthritis Rheumatol 66 (1), 49-59. doi: 10.1002/art.38142.

Lin, S., Pan, L., Guo, S., Wu, J., Jin, L., Wang, J.C., et al. (2013). Prognostic role of microRNA-181a/b in hematological malignancies: a meta-analysis. PLoS One 8 (3), e59532. doi:

10.1371/journal.pone.0059532.

Liu, C., Pan, A., Chen, X., Tu, J., Xia, X., and Sun, L. (2019a). MiR-5571-3p and miR-135b-5p, derived from analyses of microRNA profile sequencing, correlate with increased disease risk and activity of rheumatoid arthritis. Clin Rheumatol 38 (6), 1753-1765. doi: 10.1007/s10067-018-04417-w.

Liu, J., Fei, D., Xing, J., and Du, J. (2017). MicroRNA-29a inhibits proliferation and induces apoptosis in rheumatoid arthritis fibroblast-like synoviocytes by repressing STAT3. Biomed Pharmacother 96, 173-181. doi: 10.1016/j.biopha.2017.09.120.

Liu, X., Ni, S., Li, C., Xu, N., Chen, W., Wu, M., et al. (2019b). Circulating microRNA-23b as a new biomarker for rheumatoid arthritis. Gene 712, 143911. doi: 10.1016/j.gene.2019.06.001.

Liu, Y., Han, Y., Qu, H., Fang, J., Ye, M., and Yin, W. (2019c). Correlation of microRNA expression profile with clinical response to tumor necrosis factor inhibitor in treating rheumatoid arthritis patients: A prospective cohort study. J Clin Lab Anal 33 (7), e22953. doi: 10.1002/jcla.22953.

Long, L., Yu, P., Liu, Y., Wang, S., Li, R., Shi, J., et al. (2013). Upregulated microRNA-155 expression in peripheral blood mononuclear cells and fibroblast-like synoviocytes in rheumatoid arthritis. Clin Dev Immunol 2013, 296139. doi: 10.1155/2013/296139.

Maeda, Y., Farina, N.H., Matzelle, M.M., Fanning, P.J., Lian, J.B., and Gravallese, E.M. (2017). Synovium-Derived MicroRNAs Regulate Bone Pathways in Rheumatoid Arthritis. J Bone Miner Res 32 (3), 461-472. doi: 10.1002/jbmr.3005.

Mallinson, D.J., Dunbar, D.R., Ridha, S., Sutton, E.R., De la Rosa, O., Dalemans, W., et al. (2017). Identification of Potential Plasma microRNA Stratification Biomarkers for Response to Allogeneic Adipose-Derived Mesenchymal Stem Cells in Rheumatoid Arthritis. Stem Cells Transl Med 6 (4), 1202-1206. doi: 10.1002/sctm.16-0356.

Meng, H.Y., Chen, L.Q., and Chen, L.H. (2020). The inhibition by human MSCs-derived miRNA-124a overexpression exosomes in the proliferation and migration of rheumatoid arthritis-related 
fibroblast-like synoviocyte cell. BMC Musculoskelet Disord 21 (1), 150. doi: 10.1186/s12891020-3159-y.

Mizoguchi, F., and Kohsaka, H. (2012). [Role of microRNA in rheumatoid arthritis]. Nihon Rinsho Meneki Gakkai Kaishi 35 (1), 69-74. doi: 10.2177/jsci.35.69.

Mookherjee, N., and El-Gabalawy, H.S. (2013). High degree of correlation between whole blood and PBMC expression levels of miR-155 and miR-146a in healthy controls and rheumatoid arthritis patients. J Immunol Methods 400-401, 106-110. doi: 10.1016/j.jim.2013.10.001.

Murata, K., Furu, M., Yoshitomi, H., Ishikawa, M., Shibuya, H., Hashimoto, M., et al. (2013). Comprehensive microRNA analysis identifies miR-24 and miR-125a-5p as plasma biomarkers for rheumatoid arthritis. PLoS One 8 (7), e69118. doi: 10.1371/journal.pone.0069118.

Nakamachi, Y., Kawano, S., Takenokuchi, M., Nishimura, K., Sakai, Y., Chin, T., et al. (2009). MicroRNA-124a is a key regulator of proliferation and monocyte chemoattractant protein 1 secretion in fibroblast-like synoviocytes from patients with rheumatoid arthritis. Arthritis Rheum 60 (5), 1294-1304. doi: 10.1002/art.24475.

Niimoto, T., Nakasa, T., Ishikawa, M., Okuhara, A., Izumi, B., Deie, M., et al. (2010). MicroRNA-146a expresses in interleukin-17 producing T cells in rheumatoid arthritis patients. BMC Musculoskelet Disord 11, 209. doi: 10.1186/1471-2474-11-209.

Oka, S., Furukawa, H., Shimada, K., Hashimoto, A., Komiya, A., Fukui, N., et al. (2017). Plasma miRNA expression profiles in rheumatoid arthritis associated interstitial lung disease. BMC Musculoskelet Disord 18 (1), 21. doi: 10.1186/s12891-017-1389-4.

Okada, Y., Wu, D., Trynka, G., Raj, T., Terao, C., Ikari, K., et al. (2014). Genetics of rheumatoid arthritis contributes to biology and drug discovery. Nature 506 (7488), 376-381. doi: 10.1038/nature12873.

Okamoto, K., Nakashima, T., Shinohara, M., Negishi-Koga, T., Komatsu, N., Terashima, A., et al. (2017). Osteoimmunology: The Conceptual Framework Unifying the Immune and Skeletal Systems. Physiol Rev 97 (4), 1295-1349. doi: 10.1152/physrev.00036.2016.

Ormseth, M.J., Solus, J.F., Sheng, Q., Ye, F., Song, H., Wu, Q., et al. (2020a). The Endogenous Plasma Small RNAome of Rheumatoid Arthritis. ACR Open Rheumatol 2 (2), 97-105. doi: 10.1002/acr2.11098.

Ormseth, M.J., Solus, J.F., Sheng, Q., Ye, F., Wu, Q., Guo, Y., et al. (2020b). Development and Validation of a MicroRNA Panel to Differentiate Between Patients with Rheumatoid Arthritis or Systemic Lupus Erythematosus and Controls. J Rheumatol 47 (2), 188-196. doi: 10.3899/jrheum.181029.

Ormseth, M.J., Solus, J.F., Vickers, K.C., Oeser, A.M., Raggi, P., and Stein, C.M. (2015). Utility of Select Plasma MicroRNA for Disease and Cardiovascular Risk Assessment in Patients with Rheumatoid Arthritis. J Rheumatol 42 (10), 1746-1751. doi: 10.3899/jrheum.150232.

Ouboussad, L., Hunt, L., Hensor, E.M.A., Nam, J.L., Barnes, N.A., Emery, P., et al. (2017). Profiling microRNAs in individuals at risk of progression to rheumatoid arthritis. Arthritis Res Ther 19 (1), 288. doi: 10.1186/s13075-017-1492-9.

Perez-Sanchez, C., Cecchi, I., Barbarroja, N., Patino-Trives, A.M., Luque-Tevar, M., Perez-Sanchez, L., et al. (2019). Early restoration of immune and vascular phenotypes in systemic lupus erythematosus and rheumatoid arthritis patients after B cell depletion. J Cell Mol Med 23 (9), 6308-6318. doi: 10.1111/jcmm.14517.

Raj Christian, S.D., Thirugnanasambantham, K., Islam, M.I.H., Sudalaimuthu, M.K., Sundaram, S., Ashok, G., et al. (2019). Identification of Expressed miRNAs in Human Rheumatoid Arthritis Using Computational Approach - Discovery of a New miR-7167 from Human. Microrna 8 (2), 147-154. doi: 10.2174/2211536608666181204111438.

Rana, A.K., Li, Y., Dang, Q., and Yang, F. (2018). Monocytes in rheumatoid arthritis: Circulating precursors of macrophages and osteoclasts and, their heterogeneity and plasticity role in RA pathogenesis. Int Immunopharmacol 65, 348-359. doi: 10.1016/j.intimp.2018.10.016. 
Ren, B., Liu, J., Wu, K., Zhang, J., Lv, Y., Wang, S., et al. (2019). TNF-alpha-elicited miR-29b potentiates resistance to apoptosis in peripheral blood monocytes from patients with rheumatoid arthritis. Apoptosis 24 (11-12), 892-904. doi: 10.1007/s10495-019-01567-3.

Romo-Garcia, M.F., Bastian, Y., Zapata-Zuniga, M., Macias-Segura, N., Castillo-Ortiz, J.D., LaraRamirez, E.E., et al. (2019). Identification of putative miRNA biomarkers in early rheumatoid arthritis by genome-wide microarray profiling: A pilot study. Gene 720, 144081. doi: 10.1016/j.gene.2019.144081.

Senousy, M.A., Helmy, H.S., Fathy, N., Shaker, O.G., and Ayeldeen, G.M. (2019). Association of MTMR3 rs12537 at miR-181a binding site with rheumatoid arthritis and systemic lupus erythematosus risk in Egyptian patients. Sci Rep 9 (1), 12299. doi: 10.1038/s41598-019-48770-5.

Shaker, O.G., El Boghdady, N.A., and El Sayed, A.E. (2018). Association of MiRNA-146a, MiRNA-499, IRAK1 and PADI4 Polymorphisms with Rheumatoid Arthritis in Egyptian Population. Cell Physiol Biochem 46 (6), 2239-2249. doi: 10.1159/000489592.

Shi, D.L., Shi, G.R., Xie, J., Du, X.Z., and Yang, H. (2016). MicroRNA-27a Inhibits Cell Migration and Invasion of Fibroblast-Like Synoviocytes by Targeting Follistatin-Like Protein 1 in Rheumatoid Arthritis. Mol Cells 39 (8), 611-618. doi: 10.14348/molcells.2016.0103.

Smigielska-Czepiel, K., van den Berg, A., Jellema, P., van der Lei, R.J., Bijzet, J., Kluiver, J., et al. (2014). Comprehensive analysis of miRNA expression in T-cell subsets of rheumatoid arthritis patients reveals defined signatures of naive and memory Tregs. Genes Immun 15 (2), 115-125. doi: 10.1038/gene.2013.69.

Sode, J., Krintel, S.B., Carlsen, A.L., Hetland, M.L., Johansen, J.S., Horslev-Petersen, K., et al. (2018). Plasma MicroRNA Profiles in Patients with Early Rheumatoid Arthritis Responding to Adalimumab plus Methotrexate vs Methotrexate Alone: A Placebo-controlled Clinical Trial. $J$ Rheumatol 45 (1), 53-61. doi: 10.3899/jrheum.170266.

Stanczyk, J., Ospelt, C., Karouzakis, E., Filer, A., Raza, K., Kolling, C., et al. (2011). Altered expression of microRNA-203 in rheumatoid arthritis synovial fibroblasts and its role in fibroblast activation. Arthritis Rheum 63 (2), 373-381. doi: 10.1002/art.30115.

Stanczyk, J., Pedrioli, D.M., Brentano, F., Sanchez-Pernaute, O., Kolling, C., Gay, R.E., et al. (2008). Altered expression of MicroRNA in synovial fibroblasts and synovial tissue in rheumatoid arthritis. Arthritis Rheum 58 (4), 1001-1009. doi: 10.1002/art.23386.

Tang, X., Wang, J., Xia, X., Tian, J., Rui, K., Xu, H., et al. (2019). Elevated expression of ciRS-7 in peripheral blood mononuclear cells from rheumatoid arthritis patients. Diagn Pathol 14 (1), 11. doi: 10.1186/s13000-019-0783-7.

Tolboom, T.C., Pieterman, E., van der Laan, W.H., Toes, R.E., Huidekoper, A.L., Nelissen, R.G., et al. (2002). Invasive properties of fibroblast-like synoviocytes: correlation with growth characteristics and expression of MMP-1, MMP-3, and MMP-10. Ann Rheum Dis 61 (11), 975-980. doi: 10.1136/ard.61.11.975.

Toraih, E.A., Ismail, N.M., Toraih, A.A., Hussein, M.H., and Fawzy, M.S. (2016). Precursor miR-499a Variant but not miR-196a2 is Associated with Rheumatoid Arthritis Susceptibility in an Egyptian Population. Mol Diagn Ther 20 (3), 279-295. doi: 10.1007/s40291-016-0194-3.

Trenkmann, M., Brock, M., Gay, R.E., Michel, B.A., Gay, S., and Huber, L.C. (2013). Tumor necrosis factor alpha-induced microRNA-18a activates rheumatoid arthritis synovial fibroblasts through a feedback loop in NF-kappaB signaling. Arthritis Rheum 65 (4), 916-927. doi: 10.1002/art.37834.

Tseng, C.C., Wu, L.Y., Tsai, W.C., Ou, T.T., Wu, C.C., Sung, W.Y., et al. (2019). Differential Expression Profiles of the Transcriptome and miRNA Interactome in Synovial Fibroblasts of Rheumatoid Arthritis Revealed by Next Generation Sequencing. Diagnostics (Basel) 9 (3). doi: 10.3390/diagnostics9030098. 
van Hamburg, J.P., and Tas, S.W. (2018). Molecular mechanisms underpinning T helper 17 cell heterogeneity and functions in rheumatoid arthritis. $J$ Autoimmun 87, 69-81. doi: 10.1016/j.jaut.2017.12.006.

Wang, L., Wang, C., Jia, X., and Yu, J. (2018a). Circulating Exosomal miR-17 Inhibits the Induction of Regulatory T Cells via Suppressing TGFBR II Expression in Rheumatoid Arthritis. Cell Physiol Biochem 50 (5), 1754-1763. doi: 10.1159/000494793.

Wang, W., Zhang, Y., Zhu, B., Duan, T., Xu, Q., Wang, R., et al. (2015). Plasma microRNA expression profiles in Chinese patients with rheumatoid arthritis. Oncotarget 6 (40), 42557-42568. doi: 10.18632/oncotarget.6449.

Wang, X., Tang, K., Wang, Y., Chen, Y., Yang, M., Gu, C., et al. (2019a). Elevated microRNA1455p increases matrix metalloproteinase 9 by activating the nuclear factorkappaB pathway in rheumatoid arthritis. Mol Med Rep 20 (3), 2703-2711. doi: 10.3892/mmr.2019.10499.

Wang, X.Y., Wang, H.L., Mao, X., Li, G.Y., Guo, Q.Y., Li, W.J., et al. (2019b). [Identification of biomarkers to response of Tripterygium Glycosides Tablets acting on rheumatoid arthritis by integrating transcriptional data mining and biomolecular network analysis]. Zhongguo Zhong Yao Za Zhi 44 (16), 3415-3422. doi: 10.19540/j.cnki.cjcmm.20181031.001.

Wang, Y., Dai, L., Wu, H., Zhang, Z.R., Wang, W.Y., Fu, J., et al. (2018b). Novel anti-inflammatory target of geniposide: Inhibiting Itgbeta1/Ras-Erk1/2 signal pathway via the miRNA-124a in rheumatoid arthritis synovial fibroblasts. Int Immunopharmacol 65, 284-294. doi: 10.1016/j.intimp.2018.09.049.

Wangyang, Y., Yi, L., Wang, T., Feng, Y., Liu, G., Li, D., et al. (2018). MiR-199a-3p inhibits proliferation and induces apoptosis in rheumatoid arthritis fibroblast-like synoviocytes via suppressing retinoblastoma 1. Biosci Rep 38 (6). doi: 10.1042/BSR20180982.

Wu, J., Fan, W., Ma, L., and Geng, X. (2018). miR-708-5p promotes fibroblast-like synoviocytes' cell apoptosis and ameliorates rheumatoid arthritis by the inhibition of Wnt3a/beta-catenin pathway. Drug Des Devel Ther 12, 3439-3447. doi: 10.2147/DDDT.S177128.

Xie, L., and Xu, J. (2018). Role of MiR-98 and Its Underlying Mechanisms in Systemic Lupus Erythematosus. J Rheumatol 45 (10), 1397-1405. doi: 10.3899/jrheum.171290.

Xie, Q., Wei, M., Zhang, B., Kang, X., Liu, D., Zheng, W., et al. (2018). MicroRNA33 regulates the NLRP3 inflammasome signaling pathway in macrophages. Mol Med Rep 17 (2), 3318-3327. doi: 10.3892/mmr.2017.8224.

Xu, K., Xu, P., Yao, J.F., Zhang, Y.G., Hou, W.K., and Lu, S.M. (2013). Reduced apoptosis correlates with enhanced autophagy in synovial tissues of rheumatoid arthritis. Inflamm Res 62 (2), 229-237. doi: 10.1007/s00011-012-0572-1.

Yan, S., Wang, P., Wang, J., Yang, J., Lu, H., Jin, C., et al. (2019). Long Non-coding RNA HIX003209 Promotes Inflammation by Sponging miR-6089 via TLR4/NF-kappaB Signaling Pathway in Rheumatoid Arthritis. Front Immunol 10, 2218. doi: 10.3389/fimmu.2019.02218.

Yang, B., Chen, J., Li, Y., Zhang, J., Li, D., Huang, Z., et al. (2012). Association of polymorphisms in premiRNA with inflammatory biomarkers in rheumatoid arthritis in the Chinese Han population. Hum Immunol 73 (1), 101-106. doi: 10.1016/j.humimm.2011.10.005.

Yang, B., Zhang, J.L., Shi, Y.Y., Li, D.D., Chen, J., Huang, Z.C., et al. (2011). Association study of single nucleotide polymorphisms in pre-miRNA and rheumatoid arthritis in a Han Chinese population. Mol Biol Rep 38 (8), 4913-4919. doi: 10.1007/s11033-010-0633-x.

Yang, G., Wu, D., Zeng, G., Jiang, O., Yuan, P., Huang, S., et al. (2015). Correlation between miR-126 expression and DNA hypomethylation of CD4+ T cells in rheumatoid arthritis patients. Int J Clin Exp Pathol 8 (8), 8929-8936. 
Yang, X.K., Li, P., Zhang, C., Leng, R.X., Li, S., Liu, J., et al. (2017). Association between IRAK1 rs3027898 and miRNA-499 rs3746444 polymorphisms and rheumatoid arthritis : A case control study and meta-analysis. Z Rheumatol 76 (7), 622-629. doi: 10.1007/s00393-016-0169-0.

Yu, S., Lu, Y., Zong, M., Tan, Q., and Fan, L. (2019). Hypoxia-induced miR-191-C/EBPbeta signaling regulates cell proliferation and apoptosis of fibroblast-like synoviocytes from patients with rheumatoid arthritis. Arthritis Res Ther 21 (1), 78. doi: 10.1186/s13075-019-1861-7.

Yue, J., Lau, T.C.K., Griffith, J.F., Xu, J., Xiao, F., Shi, L., et al. (2019). Circulating miR-99b-5p as a novel predictor of erosion progression on high-resolution peripheral quantitative computed tomography in early rheumatoid arthritis: A prospective cohort study. Int J Rheum Dis 22 (9), 1724-1733. doi: 10.1111/1756-185X.13644.

Zhang, P., Wang, J., Lu, T., Wang, X., Zheng, Y., Guo, S., et al. (2015). miR-449b rs10061133 and miR4293 rs 12220909 polymorphisms are associated with decreased esophageal squamous cell carcinoma in a Chinese population. Tumour Biol 36 (11), 8789-8795. doi: 10.1007/s13277-0153422-2.

Zhang, Y., Wang, H., Mao, X., Guo, Q., Li, W., Wang, X., et al. (2018). A Novel Circulating miRNABased Model Predicts the Response to Tripterysium Glycosides Tablets: Moving Toward ModelBased Precision Medicine in Rheumatoid Arthritis. Front Pharmacol 9, 378. doi: 10.3389/fphar.2018.00378.

Zheng, J., Zhu, L., Iok In, I., Chen, Y., Jia, N., and Zhu, W. (2020). Bone marrow-derived mesenchymal stem cells-secreted exosomal microRNA-192-5p delays inflammatory response in rheumatoid arthritis. Int Immunopharmacol 78, 105985. doi: 10.1016/j.intimp.2019.105985.

Zhou, Q., Haupt, S., Kreuzer, J.T., Hammitzsch, A., Proft, F., Neumann, C., et al. (2015a). Decreased expression of miR-146a and miR-155 contributes to an abnormal Treg phenotype in patients with rheumatoid arthritis. Ann Rheum Dis 74 (6), 1265-1274. doi: 10.1136/annrheumdis-2013-204377.

Zhou, X., Zhu, J., Zhang, H., Zhou, G., Huang, Y., and Liu, R. (2015b). Is the microRNA-146a (rs2910164) polymorphism associated with rheumatoid arthritis? Association of microRNA-146a (rs2910164) polymorphism and rheumatoid arthritis could depend on gender. Joint Bone Spine 82 (3), 166-171. doi: 10.1016/j.jbspin.2014.12.009.

Zhu, X., Wu, L., Mo, X., Xia, W., Guo, Y., Wang, M., et al. (2020). Identification of PBMC-expressed miRNAs for rheumatoid arthritis. Epigenetics 15 (4), 386-397. doi:

10.1080/15592294.2019.1676613.

\section{Figure Legends}

Figure 1. miRNA based regulatory network in rheumatoid arthritis. We extract all the regulatory network from the included studies and constructed the regulator network based on Cytoscape. We can find numerous studies are focusing on TNF-a and cytokines while some other studies focusing on epigenetic regulation and inflammatory pathways 


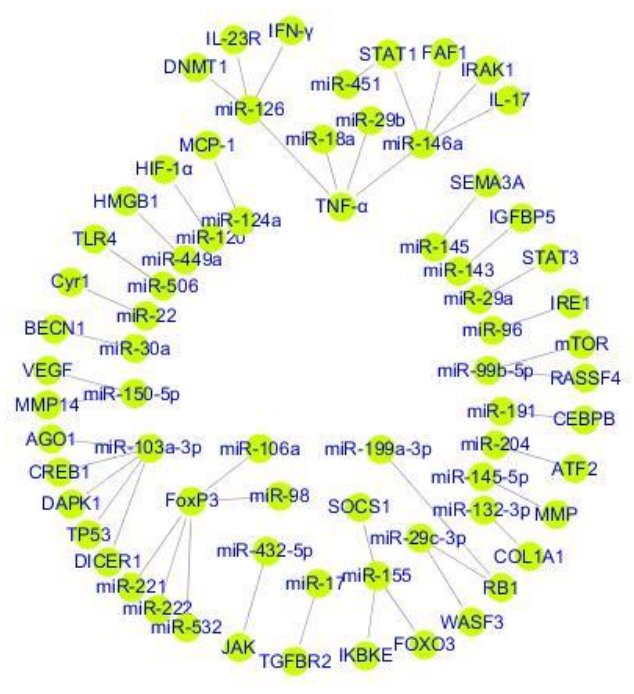

